\section{Fatigue and TSH Levels in Hypothyroid Patients} \\ Boutros El-Haddad, M.D. ${ }^{1}$, Issam El Bizri, M.D. ${ }^{2}$, K. James Kallail, Ph.D. ${ }^{1}$, \\ Rosalee E. Zackula, M.A. ${ }^{3}$, and Jan M. Hoffman, M.D. ${ }^{1,4}$ \\ ${ }^{1}$ University of Kansas School of Medicine-Wichita Department of Internal Medicine \\ ${ }^{2}$ Huggins Hospital, Wolfeboro, NH \\ ${ }^{3}$ University of Kansas School of Medicine-Wichita Office of Research \\ ${ }^{4}$ Via Christi Clinic Center for Diabetes and Endocrinology, Wichita, KS
}

\begin{abstract}
Background. Fatigue is often the complaint that initiates thyroid function investigation. Most available data related to fatigue and hypothyroidism involved patients with subclinical hypothyroidism where fatigue was not the primary outcome. This study investigated the association between TSH levels and fatigue and if there was a target TSH interval that was associated with lower incidence of fatigue in patients with hypothyroidism.

Methods. An analytic, retrospective cohort study design assessed the relationship between TSH levels and fatigue. All adult patients at one endocrinology clinic who were diagnosed with hypothyroidism between January 1, 2006 and December 31, 2007 were included. Diagnoses were confirmed by biochemical testing. Data were abstracted from the clinic's electronic medical record. Fatigue status was self-reported by the subject during examination by the endocrinologist. Fatigue status and TSH levels were obtained twice: at diagnosis and during the first follow-up visit.

Results. A total of 135 patients met the inclusion criteria. After treatment, all patients had reductions in TSH levels. Those subjects reporting relief from fatigue tended to be males ( $\mathrm{p}=$ 0.003), had lower TSH levels at follow-up ( $<<0.001)$, had larger TSH differences from baseline $(\mathrm{p}=0.007)$, and had a primary diagnosis of acquired hypothyroidism $(\mathrm{p}<0.001)$. Females were 2.9 times more likely to report persistent fatigue than males. Patients with primary diagnosis of thyroiditis were 3 times more likely to report persistent fatigue than those with acquired hypothyroidism.
\end{abstract}

Conclusions. The observed relief from fatigue after treatment correlated with a higher TSH reduction compared to patients with persistent fatigue. It was unclear if fatigue relief was related to the level of TSH reduction (TSH difference) or to a lower absolute TSH level reached after treatment.

KJM 2012; 5(2):51-57.

\section{Introduction}

Hypothyroidism is a common condition affecting $4.6 \%$ of the US population. ${ }^{1}$ Hypothyroidism manifests different levels of severity and presents with a wide interindividual range of clinical and biochemical signs and symptoms. ${ }^{2}$ Most of the clinical manifestations of hypothyroidism are related to the lack of thyroid hormone. The lack of thyroid hormone results in a generalized slowing of metabolic processes leading to fatigue, cold intolerance, weight gain, generalized slowing, and bradycardia.,4 Advances and improvements in the diagnosis of hypothyroidism have meant that a larger number of patients are being detected with the condition. ${ }^{5}$ Endocrinologists titrate thyroid hormone replacement based on thyroid stimulating hormone (TSH) levels. Despite the improvement in laboratory diagnosis, follow-up, and 
monitoring, patient's satisfaction has not improved. ${ }^{6}$

Fatigue and obesity are often the complaints that initiates thyroid function investigation. ${ }^{3,4}$ Fatigue is reported in 21\% of patient encounters in primary care. $^{7}$ Testing in the fatigued patient is increasing, although the yield is low, particularly with thyroid testing. Fatigue poses a diagnostic challenge for clinicians with its extensive differential diagnosis. Fatigue symptoms usually begin long before presentation and often remain after treatment. Thyroid function testing is recommended only when symptoms of fatigue are prolonged and debilitating. ${ }^{4}$

Most available data related to fatigue and hypothyroidism involved patients with subclinical hypothyroidism where fatigue was not the primary outcome., ${ }^{2,8,9}$ For example, symptoms, including fatigue, in patients with subclinical hypothyroidism improved with hormone replacement. ${ }^{2}$ Two clinical scores assessing symptoms and signs of hypothyroidism (Billewicz and Zulewski scores) improved significantly. However, no specific interval or target for the TSH level was suggested as a result of these scores. Another randomized trial concluded that there were no clinically relevant benefits from six months of thyroxine treatment in women with mild subclinical hypothyroidism. ${ }^{2}$

The aim of treatment for hypothyroidism is to restore the patient's TSH value to normal. Some patients may feel better only when their TSH level below $2.5 \mathrm{mU} / \mathrm{l}$, the lower half of the reference range. ${ }^{4}$ In fact, quality of life measures (including fatigue) are reduced distinctly in thyroid cancer patients undergoing thyroid hormone withdrawal. ${ }^{10}$ Thus, low TSH levels are important for symptom reduction in hypothyroid patients. However, the target TSH that may improve symptoms of fatigue is unclear.
The purpose of this study was to investigate the association between $\mathrm{TSH}$ levels and fatigue and to investigate if there is a target TSH range that was associated with lower incidence of fatigue in patients with hypothyroidism. The research question was: Is there an association between fatigue and TSH levels in patients with hypothyroidism on hormonal replacement therapy? It was hypothesized that a specific range of TSH will be associated with less fatigue.

\section{Methods}

Study design. An analytic, retrospective cohort study design was used to assess the relationship between TSH levels and fatigue. The study was approved by the university Institutional Review Board.

Setting and data source. The study took place at an endocrinology clinic in Wichita, Kansas. Clinic staff provided names of all adult patients who were diagnosed with a type of hypothyroidism (by $\mathrm{JH}$ ) between January 1, 2006 and December 31, 2007. One investigator (IEB) reviewed charts of eligible participants and diagnoses were confirmed by biochemical testing. Data were abstracted from the clinic's electronic medical record.

Participants. Eligible participants were adult clinic patients, aged 18 or over, with diagnoses of primary hypothyroidism according to the International Classification of Diseases, $9^{\text {th }}$ Revision (ICD-9). Specifically, clinic staff identified patients with the following ICD-9 codes: 244: Acquired hypothyroidism (Ablation), 245: Thyroiditis (Autoimmune) and 244.9: Unspecified hypothyroidism (NOS). Inclusion criteria were: a) documented reported fatigue prior to treatment, b) documented fatigue status at three-month follow-up, c) hormone replacement therapy, and d) record of TSH levels from both initial and three-month follow-up visits. Exclusion 
criteria were: a) patients with other known causes for fatigue at the time of diagnosis (i.e., cancer, including thyroid cancer, uncontrolled diabetes, sleep apnea, advanced chronic obstructive pulmonary disease, fibromyalgia, clinical depression, among others), b) patients on T3 (liothyronine), and c) those who obtained biochemical testing at other facilities.

$\underline{\text { Variables. }}$. The outcome of interest was fatigue status following hormone replacement therapy. Fatigue status was selfreported as present or absent by the subject during examination and interview by the endocrinologist. Fatigue status and TSH levels were obtained twice: at diagnosis (baseline) and during the first follow-up visit, usually at 3 months (post-treatment). Fatigue status was identified as those patients reporting fatigue at follow-up (Persistent Fatigue) and those reporting no fatigue (Relief from Fatigue). In an effort to control for information bias associated with self-reported fatigue, only those patients with a documented complaint of fatigue prior to treatment were included in the analyses. Reduction in TSH level was calculated by subtracting follow-up levels from baseline (TSH difference). Predictor variables were age, body mass index (BMI), and TSH difference. Potential confounders/ effect modifiers (i.e., sex, type of primary diagnosis, and BMI by TSH difference interaction) were assessed with multivariable analysis.

Statistical methods. Sample size was determined by the number of patients in the clinic who met inclusion/exclusion criteria during the study period. Quantitative variables (age, BMI, and TSH difference) were assessed for normality, while categorical variables were summarized by frequency and/or proportions. Descriptive analysis was conducted for patient characteristics and TSH level at baseline and at follow-up. Univariate analyses (Kolmo-
gorov-Smirnov, Chi-square or Fisher's Exact Test, risk estimates, Mann-Whitney Test) were conducted to compare Persistent Fatigue with Relief from Fatigue groups. Logistic regression models (Generalized Linear Models: binomial probability distribution with logit link function) that incorporated TSH difference, including interactions, were explored. Criteria for best model was based on goodness-of-fit measures [i.e., Deviance (closest value to one), AIC, and BIC (small-is-better form)]. Analyses were conducted in PASW Statistics 18 (SPSS), IBM Corp., Somers, NY.

\section{Results}

Eligible participants included 183 patients identified with one of the three types of hypothyroidism. Of those, 135 (74\%) met the inclusion criteria and were included in the final analysis. Table 1 summarizes patient demographics, TSH levels, diagnoses, and univariate analyses by fatigue status. Data were sparse and test results of the continuous variables (age, BMI, TSH levels) by Fatigue Status using the Kolmogorov-Smirnov tests were inconclusive. However, histograms revealed a potential non-Gaussian distribution, therefore, non-parametric Mann-Whitney exact tests were conducted. Likewise, Fisher's exact tests were reported for gender and diagnosis. Results showed significant differences for gender, TSH follow-up, TSH difference, and diagnosis.

Those subjects reporting relief from fatigue tended to be males more so than females ( $p=0.003)$, had lower TSH levels at follow-up ( $p<0.001)$, had larger TSH difference ( $p=0.007)$, and had a primary diagnosis of post ablative hypothyroidism (p $<0.001$ ). Crude risk estimates showed that females were 2.9 times more likely to report persistent fatigue than males. Similarly, patients with primary diagnosis of 
Table 1. Characteristics of adults with hypothyroidism presenting with fatigue.

\begin{tabular}{|c|c|c|c|c|}
\hline \multicolumn{2}{|r|}{$\begin{array}{l}\text { Relief from } \\
\text { Fatigue }\end{array}$} & $\begin{array}{l}\text { Persistent } \\
\text { Fatigue }\end{array}$ & & \\
\hline Gender (\%) & $\mathrm{n}=87$ & $\mathrm{n}=48$ & $\mathrm{p}$ & $\mathrm{RR}_{\text {crude }}$ \\
\hline Female & $58(66.7)$ & 43 (89.6) & 0.003 & 2.9 \\
\hline Male & 29 (33.3) & $5(10.4)$ & & ref \\
\hline \multicolumn{5}{|l|}{ Diagnosis (\%) } \\
\hline $\begin{array}{l}\text { Acquired (post ablation) } \\
\text { Hypothyroidism }\end{array}$ & 69 (79.3) & 18 (37.5) & $<0.001$ & ref \\
\hline Thyroiditis & $18(20.7)$ & $30(62.5)$ & & 3.0 \\
\hline Unspecified Hypothyroidism & -- & -- & & \\
\hline \multicolumn{5}{|c|}{ Median (range) } \\
\hline Age & $51.0(33,74)$ & $54.5(32.0,73.0)$ & 0.793 & \\
\hline BMI & $\begin{array}{l}27.0(19.0, \\
36.0)\end{array}$ & $28.0(19.0,36.0)$ & 0.33 & \\
\hline TSH baseline & $10.8(6.2,16.0)$ & $10.4(6.2,14.8)$ & 0.971 & \\
\hline TSH follow-up & $1.0(0.5,7.4)$ & $1.85(0.3,8.6)$ & $<0.001$ & \\
\hline TSH difference & $9.5(3.2,14.6)$ & $7.9(2.6,13.7)$ & 0.007 & \\
\hline
\end{tabular}

thyroiditis were three times more likely to report persistent fatigue than those with post ablative hypothyroidism. The conflicting results for gender by diagnosis by fatigue status were interesting, such that, of the 30 females diagnosed with thyroiditis, 90\% reported persistent fatigue, whereas of the 18 males with thyroiditis, $83.3 \%$ reported relief from fatigue, although data were sparse.

Figure 1 demonstrates the relationship between fatigue status and TSH levels at baseline and follow-up, with regard to primary diagnosis. With the exception of two female patients (top: age 46 with BMI = 21, bottom: age 63, BMI =34), a linear (and lower) trend was observed for relief from fatigue compared with persistent fatigue.

Table 2 shows results from the final Generalized Linear Model. Goodness of fit measures were Deviance $=1.434$, AIC $=$ 129.76, $\mathrm{BIC}=141.38$, with gender, diagnosis, and TSH difference included in the model. All were statistically significant.
Greater reductions in TSH level were associated with relief from fatigue, while controlling for the effects of gender and diagnosis $\left(\mathrm{RR}_{\mathrm{adj}}=1.22, p=0.033\right)$. In addition, results showed that patients diagnosed with acquired (post ablation) hypothyroidism were more than 14 times as likely to report relief from fatigue than those diagnosed with thyroiditis.

More than $51 \%$ of patients were diagnosed with acquired hypothyroidism (69 out of 135, and of these 55 were female); moreover, the greatest reduction in TSH levels, as measured by median TSH difference, were observed within this group (see Figure 2).

\section{Discussion}

The study showed that thyroid hormonal replacement was associated with improvement in reported fatigue in the majority of patients. This observation was expected, as most of the symptoms of hypothyroidism are related to thyroid hormone deficiency. 


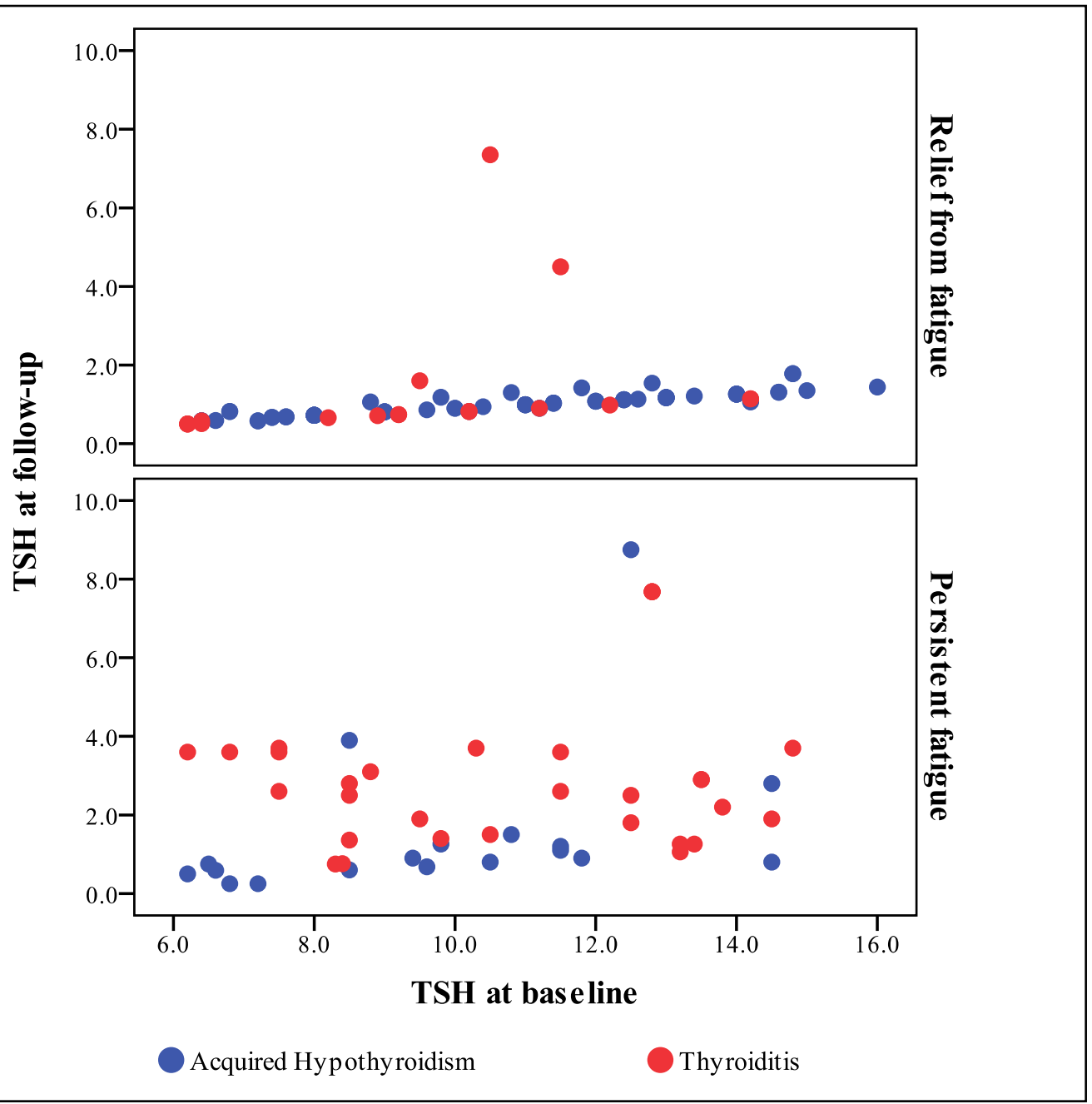

Figure 1. Relationship between fatigue status, TSH levels, and diagnosis.

Table 2. Multivariable logistic regression analysis of relief from fatigue compared with persistent fatigue adjusted for gender diagnosis and reduction in TSH level (TSH difference).

\begin{tabular}{|l|c|c|c|c|}
\hline \multicolumn{3}{|c|}{} & \multicolumn{2}{c|}{$95 \%$ Wald CI } \\
\hline Gender & $\mathrm{RR}_{\mathrm{adj}}$ & $\mathrm{p}$ & Lower & Upper \\
\hline Female & & & & \\
\hline Male & 0.05 & $<0.001$ & 0.01 & 0.22 \\
\hline Diagnosis & ref & & & \\
\hline Ablation & & & & \\
\hline Autoimmune & 14.09 & $<0.001$ & 4.73 & 42.00 \\
\hline TSH difference & ref & & & \\
\hline
\end{tabular}




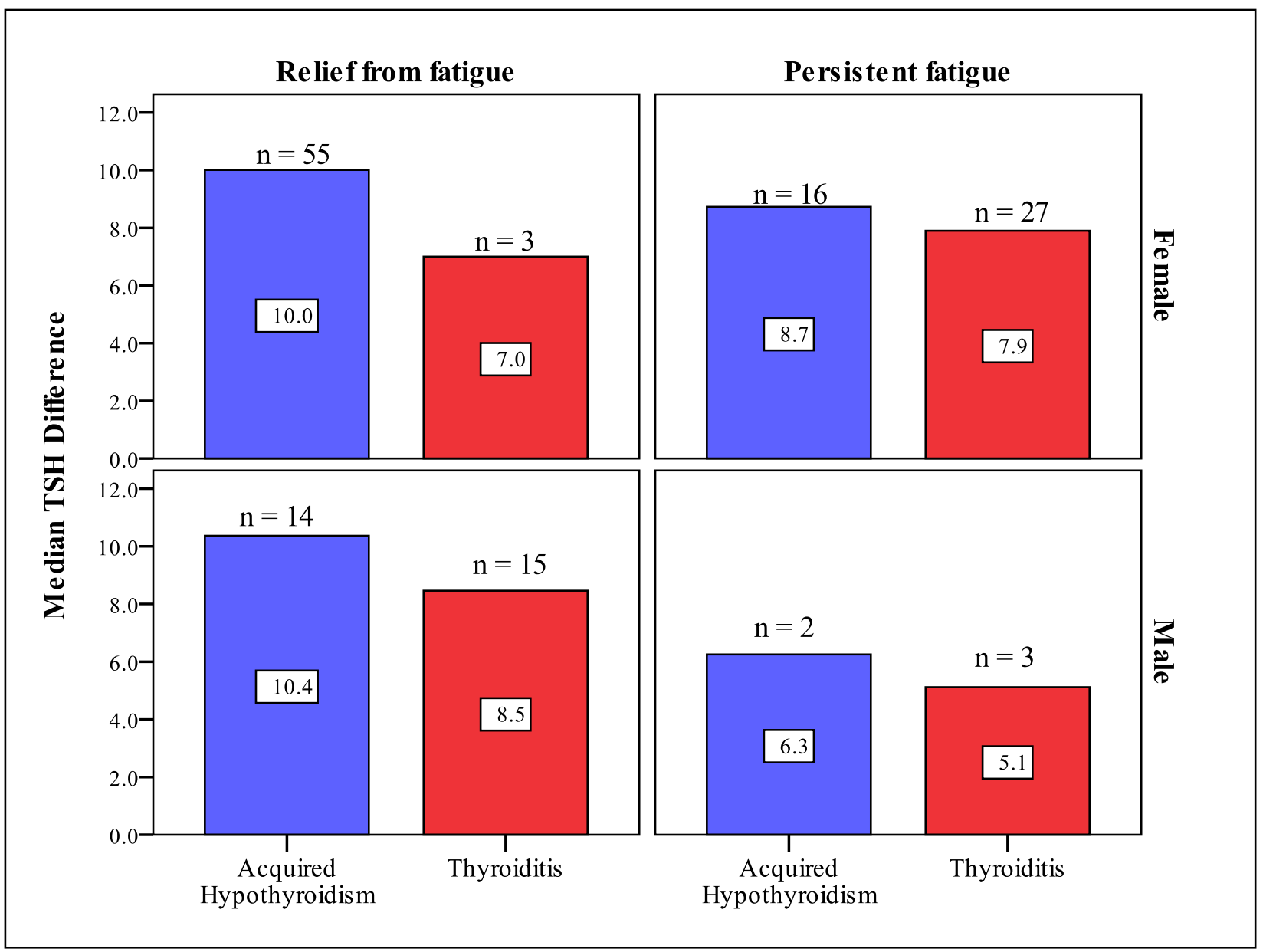

Figure 2. Fatigue status by gender, diagnosis, and median TSH difference.

Despite treatment and the decrease of TSH value, fatigue persisted in a number of patients. Interestingly, the observed relief from fatigue after treatment correlated with a higher TSH reduction compared to patients with persistent fatigue. This observation may be related to presence of a higher concentration of thyroid hormone in patients reporting improvement in fatigue, thereby resulting in more inhibition of $\mathrm{TSH}$ secretion. It was unclear if fatigue relief was related to the level of TSH reduction (TSH difference) or to a lower absolute TSH level reached after treatment.

In addition, patients with autoimmune thyroiditis were less likely to report improvement of fatigue compared to the post ablation group. The observed variability of response to treatment between patients with thyroiditis and patients with post ablation hypothyroidism could be related to the association of autoimmune thyroiditis to other autoimmune disorders that may have been clinically silent. However, groups were imbalanced and data were sparse. More than $51 \%$ of patients were diagnosed with acquired (post ablation) hypothyroidism (69 out of 135, and of these 55 were female); moreover, the greatest reduction in TSH levels, as measured by median TSH difference, were observed within this group (see Figure 2). Thus, evaluating interactions among variables were not statistically feasible. 
These results were limited in several ways. Data were obtained from one endocrinology clinic by one endocrinologist. Initial TSH levels differed widely. Fatigue was self-reported and no validated measure of fatigue was used. Fatigue was assessed only at diagnosis and the three-month follow-up. A longer follow-up is needed to assess the effect of thyroid hormonal replacement therapy on fatigue.

Despite these limitations, the study results are interesting. It showed variable

\section{References}

1 Aoki Y, Belin RM, Clicker R, Jeffries R, Phillips L, Mahaffey KR. Serum TSH and total T4 in the United States population and their association with participant characteristics: National health and Nutrition Examination Survey (NHANES 1999-2002). Thyroid 2007; 17(12):12111223. PMID: 18177256.

2 Meier C, Staub JJ, Roth CB, et al. TSHcontrolled L-thyroxine therapy reduces cholesterol levels and clinical symptoms in subclinical hypothyroidism: A double blind, placebo-controlled trial (Basel Thyroid Study). J Clin Endocrinol Metab 2001; 86(10):4860-4866. PMID: 11600 554.

${ }^{3}$ Evans KM, Flanagan DE, Wilkin TJ. Chronic fatigue: Is it endocrinology? Clin Med 2009; 9(1):34-38. PMID: 19271598.

4 Todd CH. Management of thyroid disorders in primary care: Challenges and controversies. Postgrad Med J 2009; 85(1010):655-659. PMID: 20075403.

${ }^{5}$ Unnikrishnan AG, Menon UV. Thyroid disorders in India: An epidemiological perspective. Indian $\mathrm{J}$ Endocrinol Metab 2011; 15(Suppl 2):S78-S81. PMID: 21966658.

${ }^{6}$ Kalra S, Khandelwal SK. Why are our hypothyroid patients unhappy? Is tissue hypothyroidism the answer? Indian J response to treatment by gender and by diagnosis. It is possible that female patients and patients with a diagnosis of thyroiditis are less likely to respond to conventional treatment and require a higher dose of thyroid hormonal replacement therapy. The study was not designed to answer this question. Future studies should consider a prospective cohort design that match patients on diagnosis type, then reassess the effects of gender and TSH levels on various levels of fatigue.

Endocrinol Metab 2011; 15(Suppl 2):S95S98. PMID: 21966661.

${ }^{7}$ Harrison M. Pathology testing in the tired patient: A rationale approach. Aust Fam Physician 2008; 37(11):908-910. PMID: 19037463.

${ }^{8}$ Kong WM, Sheikh MH, Lumb PJ, et al. A 6-month randomized trial of thyroxine treatment in women with mild subclinical hypothyroidism. Am J Med 2002; 112(5):348-354. PMID: 11904108.

${ }^{9}$ Iqbal A, Jorde R, Figenschau Y. Serum lipid levels in relation to serum thyroidstimulating hormone and the effect of thyroxine treatment on serum lipid levels in subjects with subclinical hypothyroidism: The Tromsø Study. J Intern Med 2006; 260(1):53-61. PMID: 16789 979.

${ }^{10}$ Tagay S, Herpertz S, Langkafel M, et al. Health-related quality of life, anxiety and depression in thyroid cancer patients under short-term hypothyroidism and TSHsuppressive levothyroxine treatment. Eur J Endocrinol 2005; 153(6):755-763. PMID: 16322380.

Keywords: hypothyroidism, fatigue, endocrinology 